\title{
Erroneously Analyzed F-Wave of Bradycardic Atrial Fibrillation as Ventricular Fibrillation for a Cardiac Arrest Patient
}

\author{
Chikara Watanabe ${ }^{a, b}$ Kento Nakajimaa, ${ }^{a}$ Kosuke Moria, \\ Masayasu Gakumazawa ${ }^{a, b}$ Naoki Nakayama ${ }^{a, c} \quad$ Makoto Kato $^{a, d}$ \\ Fumihiro Ogawa ${ }^{a, b} \quad$ Kouhei Takahashi ${ }^{a, b}$ Masayuki Iwashita ${ }^{a, b}$ \\ Takeru Abe ${ }^{b} \quad$ Ichiro Takeuchi ${ }^{a, b}$
}

${ }^{a}$ Advanced Critical Care and Emergency Center, Yokohama City University Medical Center, Yokohama, Japan; 'bepartment of Emergency Medicine, Yokohama City University School of Medicine, Yokohama, Japan; 'Department of Medical Science and Cardiorenal Medicine, Yokohama City University School of Medicine, Yokohama, Japan;

dDepartment of Surgery, Yokohama City University School of Medicine, Yokohama, Japan

\section{Keywords}

Automated external defibrillator - Atrial fibrillation · Cardiopulmonary resuscitation .

Defibrillation

\section{Abstract}

The prognosis of out-of-hospital cardiac arrest has improved in Japan due to the increasing availability of automated external defibrillators. However, priority should be placed on specificity to not perform unnecessary waveforms rather than sensitivity to perform defibrillation on necessary waveforms. This report describes a case in which the f-wave of bradycardic atrial fibrillation was erroneously analyzed and defibrillated. Health care workers should be aware that the $\mathrm{f}$-wave of bradycardic atrial fibrillation may be erroneously assessed as fine ventricular fibrillation; they should change it to the manual mode and respond as necessary. 


\section{Introduction}

With the availability of automated external defibrillators (AEDs), the prognosis of out-ofhospital cardiac arrest has improved in Japan and the US [1,2]. AEDs are presently in widespread use in Japanese communities, following recommendations by the Japan Resuscitation Council (JRC), the American Heart Association (AHA), and the Association for the Advancement of Medical Instrumentation (AAMI). When using AEDs, priority should be given to specificity to not perform unnecessary waveforms, rather than sensitivity to perform defibrillation on necessary waveforms [3-5]. In Japan, the breakdown of inappropriate AED operation was $35.4 \%$ when not defibrillating necessary defibrillation waveforms, $10.5 \%$ for defibrillation requiring unnecessary defibrillation waveforms, and 55.6\% for other factors [6, 7]. In addition, a previous study reported $4 \%$ of false positive rate in shock advice and no harmful outcome for patients. This report describes a case of AED inappropriately operated for the f-wave of atrial fibrillation lacking a QRS wave [7].

\section{Case Report and Presentation}

The patient was a 52-year-old male with an initially unknown medical history. He was estranged from his family even after their identification. He collided with a stopped passenger car while on a motorbike. After the collision, the car's driver realized the patient had no response and alerted the local emergency medical service (EMS). The EMS reached the patient 5 min later and found him in cardiopulmonary arrest (CPA). The initial waveform indicated pulseless electrical activity (PEA). An AED electric shock was applied to the ventricular fibrillation (VF)-like waveform, resulting in the return of spontaneous circulation (ROSC). However, PEA returned. The patient again went into CPA and was transported via ambulance to the Advanced Critical Care and Emergency Center at Yokohama City University Medical Center, a tertiary emergency medical center.

The EMS delivered the patient to our institute within $19 \mathrm{~min}$. Upon arrival, his initial vital signs were as follows: CPA with PEA, body temperature of $36.8^{\circ} \mathrm{C}$, with no peripheral cooling sensation. Both pupils were dilated by $6 \mathrm{~mm}$. The patient's right front forehead and lower leg were crushed. Arterial blood gas tests revealed a pH of $6.83, \mathrm{PaCO}_{2}$ of $141.0 \mathrm{~mm} \mathrm{Hg}, \mathrm{PaO}_{2}$ of $43.6 \mathrm{~mm} \mathrm{Hg}, \mathrm{FiO}_{2}$ of 1.0, bicarbonate of $22.3 \mathrm{mEq} / \mathrm{L}$, lactate of $9.8 \mathrm{mmol} / \mathrm{L}$, hemoglobin of 15.2 $\mathrm{g} / \mathrm{dL}$, and troponin-I of $0.059 \mathrm{ng} / \mathrm{mL}$.

After initial chest compressions, the patient regained ROSC. At the time, his vital signs were as follows: blood pressure of $124 / 72 \mathrm{~mm} \mathrm{Hg}$, heart rate of 66 beats/min, and respiratory rate of $21 / \mathrm{min}$, with $100 \%$ oxygen saturation on a $10 \mathrm{~L}$ reservoir mask. Oral endotracheal intubation was performed in the emergency room, and artificial respiration was started. Initially, we considered CPA due to VF, but the electrocardiogram after resuscitation showed the patient had a heart rate of 46 beats $/ \mathrm{min}$ and bradycardic atrial fibrillation, with a right bundle branch block (Fig. 1).

We then conducted chest and pelvic X-rays, which indicated no obvious rib fractures, massive hemothorax, pneumothorax, or pelvic fracture. We also performed the Focused Assessment with Sonography in Trauma (FAST). There was no liquid retention in the patient's peritoneal cavity. Computed tomography imaging (CT) revealed a vertical fracture line in the patient's second cervical vertebral body (Fig. 2a). A transthoracic cardiac ultrasound examination showed no obvious valvular diseases, but there was a diffused wall motion decline in the left ventricle. Coronary angiography demonstrated no significant coronary artery stenosis. 
Intravenous pacing was introduced in the angiography room. The patient was then admitted to the intensive care unit. Later, analysis of the AED monitor waveform in the ambulance initially indicated tachyarrhythmia/atrial fibrillation. The QRS wave was gradually lost and became bradycardic atrial fibrillation with an f-wave only.

The patient's clinical course during hospitalization was as follows: On day 1, catecholamine administration was started while pacing continued. Hypothermia therapy with a target temperature of $34^{\circ} \mathrm{C}$ was initiated against prolonged unconsciousness after cardiopulmonary resuscitation. This continued for $36 \mathrm{~h}$ until it was recovered. Bradycardic atrial fibrillation gradually improved and catecholamine was also discontinued under the administration of aluminum sulfate. Intravenous pacing was discontinued on day 5, the same day we performed magnetic resonance imaging (MRI) of the patient's neck (Fig. 2b). T2-weighted MRI imaging indicted a high signal area in the spinal cord on the dorsal side of the $2 / 3$ cervical vertebrae. The patient remained unconscious and was transferred to a hospital near his home on day 15.

\section{Discussion and Conclusion}

This report described a case in which AED was inappropriately conducted for an f-wave of atrial fibrillation lacking a QRS wave. After resuscitation, intravenous pacing was performed on bradycardic atrial fibrillation caused by cervical spinal cord injury. Hypothermia therapy was then administered due to the prolonged impairment of consciousness, but the patient did not improve and was transferred to another hospital.

We suspected that the cause of CPA was respiratory arrest and neurogenic shock associated with cervical cord injury. Initially, considering VF, an analysis of the electrocardiogram waveform revealed that defibrillation was performed on the f-wave of bradycardic atrial fibrillation. Since 2006, Japanese law has allowed EMS personnel defibrillation, tracheal intubation, and adrenaline administration in patients with CPA [8]. Analyzing the waveform at prehospital for the emergency services and judging the adaptation of defibrillation would have been difficult in this case because AED was used. We also considered sympathetic nerve excitement, hypoxia, and shock as possible causes of the patient's bradycardic atrial fibrillation.

The AED analyzes four parameters: amplitude, heart rate, conductivity, and the rate of change. It also determines the adaptation of defibrillation. In the AED analysis, defibrillation is indicated when the amplitude is $100 \mu \mathrm{V}$ or more, the heart rate is 135 beats/min or higher, there is poor conductivity, and the stability is low [9-12]. The AED prioritizes specificity (with a proportion not to defibrillate unnecessary waveforms) over sensitivity for safety [3-5]. In the present case, defibrillation was performed to $99.7 \%$ of $\mathrm{VF}$ and $81 \%$ of ventricular tachycardia, and it was set so that $100 \%$ defibrillation was not enforced by static quiescence and normal sinus rhythms. However, in this case, the f-wave was erroneously analyzed as a fine $\mathrm{VF}$, and defibrillation was in effect [13]. Typically, tachycardic atrial fibrillation gradually lost the ORS wave with high-degree atrioventricular block and slow ventricular escape rhythm, then only f-wave appeared. No escape beats occurred. A shock was delivered because isolated $\mathrm{f}$-waves were erroneously interpreted as VF.

Considering that this case involved CPA after blunt trauma, some institutions would have implemented resuscitative thoracotomy (RT). The exact CPA time in our patient was unknown, and RT was carried out in the emergency room. It is often administered to patients suspected of severe trauma, especially hemorrhagic shock or the traumatic shock of trunk trauma. However, in this case, the patient was not suspected of being cardiogenic because 
there were no obvious injuries or peripheral cold sweats in his trunk. Repeated VF caused his heartbeat to resume, so RT was not administered. In a similar case, manufacturers recognize this type of misanalysis due to baseline swings and artifacts and take measures. Routinely analyzing AED electrocardiograms is important, and we should know this type of analysis might occur.

In conclusion, this report described a case in which the f-wave of bradycardic atrial fibrillation was erroneously analyzed and defibrillated. Health care workers should be aware that the f-wave of bradycardic atrial fibrillation may be erroneously assessed as fine VF; they should change it to the manual mode and respond as necessary.

\section{Statement of Ethics}

The authors have no ethical conflicts to disclose. The Institutional Review Board at Yokohama City University approved this study with permission of a waiver of consent by patients (B170400020).

\section{Disclosure Statement}

The authors have no conflicts of interest to declare.

\section{Funding Sources}

This research did not receive any specific grant from funding agencies in the public, commercial, or not-for-profit sectors.

\section{Author Contributions}

C.W., K.N., K.M., M.G., N.N., M.K., and F.O. made substantial contributions to the study conception and design as well as the acquisition of data. C.W., K.T., M.I., T.A., and I.T. made substantial contributions to the analysis and interpretation of the data. All authors were involved in drafting the manuscript and critically revising it, and all gave approval to the final version.

\section{References}

1 Yabe M, Fujii T, Terai T, Matsumoto M. Case report on interactions between implanted pacemaker and automated external defibrillator (AED) in a patient with out-of-hospital cardiac arrest [Japanese]. Journal of Japanese Association for Acute Medicine. 2008;19:1047-51.

2 Fire and Disaster Management Agency. Overview of Emergency and Rescue, 2017. [Internet]. Tokyo. [cited 2017 Dec 19]. Available from: http://www.fdma.go.jp. Japanese.

3 Kerber RE, Becker LB, Bourland JD, Cummins RO, Hallstrom AP, Michos MB, et al. Automatic external defibrillators for public access defibrillation: recommendations for specifying and reporting arrhythmia analysis algorithm performance, incorporating new waveforms, and enhancing safety. A statement for health professionals from the American Heart Association Task Force on Automatic External Defibrillation, Subcommittee on AED Safety and Efficacy. Circulation. 1997 Mar;95(6):1677-82. 


\section{Case Reports in Acute Medicine}

Case Rep Acute Med 2019;2:25-30

DOI: $10.1159 / 000500880$

(c) 2019 The Author(s). Published by S. Karger AG, Basel www.karger.com/cra

Watanabe et al.: Erroneously Analyzed F-Wave of Bradycardic Atrial Fibrillation as Ventricular Fibrillation for a Cardiac Arrest Patient

4 Ushita M, Kohayagawa Y, Niinou N, Koshizaki M, Yamamori Y, Sasaki A, et al. A case of a patient with ventricular fibrillation, in which the AED did not assess as shockable until the 4th analysis [Japanese]. Journal of Japanese Association for Acute Medicine. 2009;20:361-6.

5 Shiramoto K, Wakamatsu H, Katsuta S, Matsumoto S, Yamada K, Harada K, et al. Manual defibrillation using an automated external defibrillator for undetected pulseless ventricular tachycardia in a patient with cardiac resynchronization therapy defibrillator [Japanese]. J Jpn Soc Intensive Care Med. 2018;25(5):379-82.

6 Kondo H, et al. A study on cases where AED malfunctions were suspected at fire fighting institutions. 2012. [Internet]. Available from: http://aed-hyogo.sakura.ne.jp/wpm/archivepdf/21-23/2_11b.pdf. Japanese.

7 Zijlstra JA, Bekkers LE, Hulleman M, Beesems SG, Koster RW. Automated external defibrillator and operator performance in out-of-hospital cardiac arrest. Resuscitation. 2017 Sep;118:140-6.

8 Ueta H, Tanaka H, Takyu H, Sagisaka R, Shirakawa T, Gotou S, et al. The effects of early adrenaline on favorable neurological outcome of out-of-hospital cardiac arrest patients [Japanese]. Journal of Japanese Society for Emergency Medicine. 2016;19:578-85.

9 Ochiai S, Saito Y, et al. Two cases of cardiopulmonary arrest patients that AED never indicated shock delivery to shockable ECG rhythm [Japanese]. Journal of Japanese Association for Acute Medicine. 2011;22(3):125-32.

10 Macdonald RD, Swanson JM, Mottley JL, Weinstein C. Performance and error analysis of automated external defibrillator use in the out-of-hospital setting. Ann Emerg Med. 2001 Sep;38(3):262-7.

11 Travers AH, Rea TD, Bobrow BJ, Edelson DP, Berg RA, Sayre MR, et al. Part 4: CPR overview: 2010 American Heart Association guidelines for cardiopulmonary resuscitation and emergency cardiovascular care. Circulation. 2010 Nov;122(18 Suppl 3):S676-84.

12 Berg RA, Hemphill R, Abella BS, Aufderheide TP, Cave DM, Hazinski MF, et al. Part 5: adult basic life support: 2010 American Heart Association guidelines for cardiopulmonary resuscitation and emergency cardiovascular care. Circulation. 2010 Nov;122(18 Suppl 3):S685-705.

13 Kishimori T, Kosuga K, Inoue T, Seki J, Inuzuka Y, Takeda S, et al. Pulseless ventricular tachycardia that AED never indicated shock delivery [Japanese]. Nippon Naika Gakkai Zasshi. 2016 Nov;105(11):2221-9.

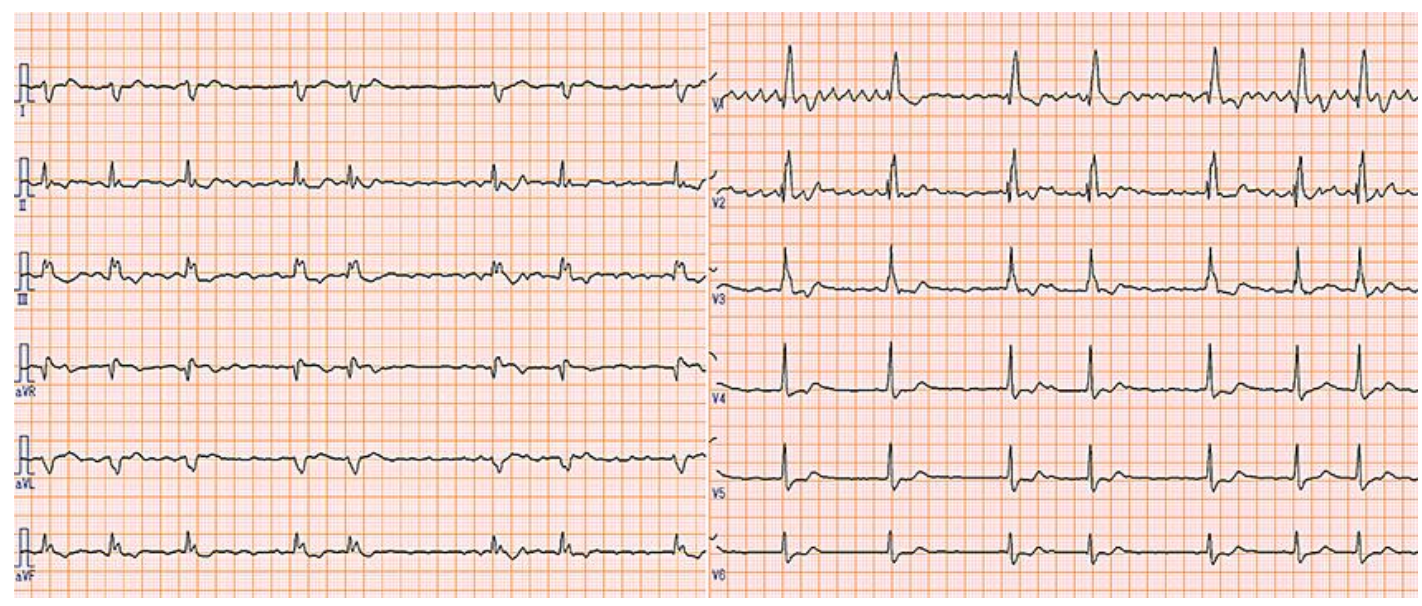

Fig. 1. Electrocardiogram indicating a heart rate of 46 beats/min, with a right leg blockage. 


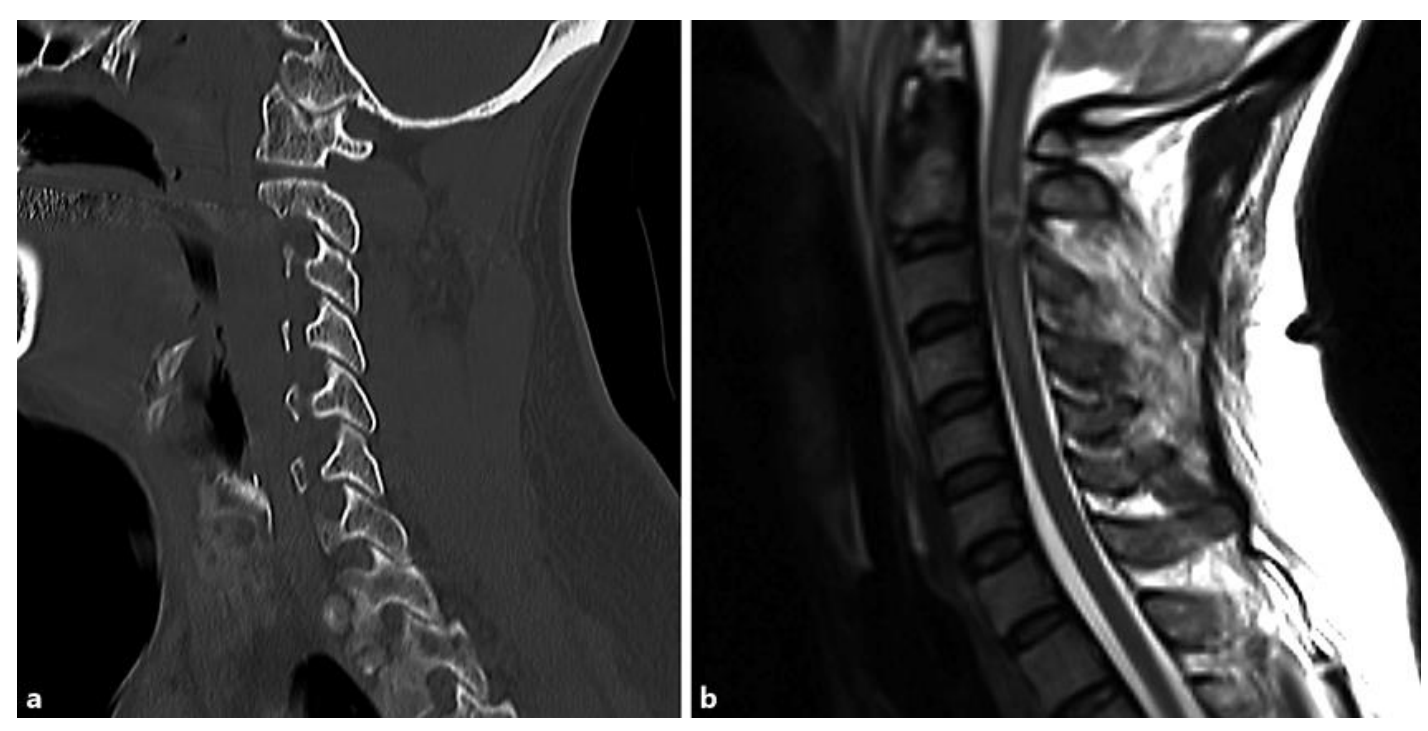

Fig. 2. a Cervical spine CT: a fracture line running longitudinally in the second cervical vertebral body. b MRI T2-weighted image: high signal area in the spinal cord between the $2 / 3$ cervical vertebrae. 